\title{
THE ORBIT SPACES OF TOTALLY DISCONNECTED GROUPS OF TRANSFORMATIONS ON MANIFOLDS
}

\author{
FRANK RAYMOND ${ }^{1}$
}

In $[2 ; 7]$ it was shown that if a $p$-adic group $A_{p}$ acts effectively as a compact group on an $n$-manifold then the integer cohomology dimension of the orbit space is $n+2$. In this note we continue the study of the orbit space of such an action. In particular we show that if we use fields for coefficients in the cohomology groups the action of a $p$-adic group closely resembles the action of a finite group.

Theorem 1 describes the orbit space of an action of a finite group. In Theorems 1 and 2 we also describe the similar situation for totally disconnected groups. As an application and corollary to Theorems 1 and 2 we may state in terms of dimension a criterion for a compact group of transformations to be a Lie group. We state the corollary here. Its proof is the last part of the paper.

COROLlaRY. Let $G$ be a compact group acting effectively on a connected separable finite dimensional (covering dimension) generalized $n$-manifold ( $n$-gm) $M$ over the integers $Z$. Let $r$ be the maximum dimension of any orbit.

(i) $G$ is a Lie group if and only if $\operatorname{dim}_{z} M / G=n-r$.

(ii) If $G$ is not a Lie group then $\operatorname{dim}_{z} M / G \geqq n-r+2$.

(iii) If $r \geqq n-2$ then $G$ is a Lie group.

(iv) If $G$ is connected and $M$ is orientable there exists an orientable $n$-gm $M / N$ over a field of characteristic 0 and a Lie group $K$ which acts on $M / N$ so that $(M / N) / K$ is naturally homeomorphic to $M / G$. ( $N$ is a totally disconnected central subgroup of $G$ and $M / N$ is its orbit space.)

That the dimension of $M / G$ is $n-r$ if $G$ is a Lie group is a theorem of Montgomery, Samelson and Zippin, and of Yang. (We shall prove the converse here.) Montgomery and Zippin have proved (iii) for $n \leqq 3$, for $r=n$, and when $M$ is $n$-dimensional Euclidean space for $r=n-1$. Bredon has proved (iii) for locally Euclidean manifolds. We shall first prove (ii). This implies (i). Moreover, the method of proof of (ii) will yield a proof of (iii).

Throughout this paper generalized manifolds are assumed to be locally orientable (see [1] or [4]). The Cech cohomology with compact supports is used throughout.

Received by the editors January 21, 1960.

1 National Science Foundation Fellow. 
I would like to express my thanks to D. Montgomery, R. S. Palais and R. F. Williams for several useful conversations concerning this paper. As in [2] there is overlap, in the free case, with the independent work of C. N. Lee.

The following lemma is easily derivable from well-known facts (see, for example, [5]).

Lemma 1. Let $M$ be a locally compact, locally connected, Hausdorff space. Let $f$ be a proper open map of $M$ onto the Hausdorff space $M^{*}$. If $U^{*}$ is an open connected set in $M^{*}$, then the components of $U=f^{-1}\left(U^{*}\right)$ are finite in number and each is mapped by $f$ onto $U^{*}$.

Lemma 2. Let $G$ be a compact group which acts on a space $M$ as above. Suppose $f$ denotes the orbit map $f: M \rightarrow M / G$; then for each component $U_{i}$ of $U=f^{-1}\left(U^{*}\right)$ there exists an open and closed subgroup $G_{i}$ of $G$ which is the largest subgroup of $G$ leaving $U_{i}$ invariant and the maps induced by $f$ map $U_{i} / G_{i}$ homeomorphically onto $U^{*}$. Moreover, if one of the $G_{i}$ is a normal subgroup of $G$ all the $G_{i}$ are identical and the order of $G / G_{i}$ is the same as the number of components of $U$.

To prove the lemma above one defines $G_{i}=\left\{g \mid g U_{i} \cap U_{i} \neq \varnothing, g \in G\right\}$. Since each $g, g \in G$, is a homeomorphism, $g U_{i}$ must actually be one of the components of $U$. Therefore, $g U_{i}=U_{i}$ if and only if $g \in G_{i}$. The rest of the lemma now follows without too much difficulty.

Lemma 3. Let $M$ and $f$ be as in Lemma 1. Suppose in addition the map $f$ is such that $f^{-1}\left(x^{*}\right)$ is totally disconnected for each $x^{*} \in M^{*}$. Let $x \in M$ and $N$ be any neighborhood containing $x$. Then there exists an open connected set $U^{*}$ containing $f(x)=x^{*}$ such that the component of $f^{-1}\left(U^{*}\right)$ containing $x$ is contained in $N$.

The next two lemmas on shuttle homomorphisms are due to Eckmann, Liao and Conner and may be found in [1, Chapter 3].

Suppose $G$ acts on the locally compact Hausdorff space $M$ and $K$ is a subgroup of $G$. Then there is an orbit map $f_{1}: M \rightarrow M / K$ and an unique map $f_{2}: M / K \rightarrow M / G$ with $f_{2} f_{1}=f ; f_{2}$ maps an orbit $K x$ of $M / K$ into the orbit $G x$ of $M / G$.

Lemma 4. If $G$ is a finite group then there is a homomorphism $u^{\prime}: H_{c}^{*}(M / K ; L) \rightarrow H_{c}^{*}(M / G ; L)$ such that $u^{\prime} f_{2}{ }^{*}$ is multiplication by order $G /$ order $K$. ( $L$ denotes any coefficient ring.)

Note the special case $K=(e)$.

If $G$ acts on a space $M$ then by $\left[H_{c}^{n}(M ; L)\right]^{G}$ we mean the set of elements of $H_{c}^{n}(M ; L)$ which are left fixed by all 


$$
g^{*}:\left(H_{c}^{n}(M ; L) \stackrel{g^{*}}{\rightarrow} H_{c}^{n}(M ; L)\right),
$$

Lemma 5. Let $L$ be a field whose characteristic is 0 or prime to the order of $G$. Then $H_{c}^{n}(M / G ; L) \approx f^{*}\left[H_{c}^{n}(M ; L)\right]^{G}$.

THEOREM 1. Let $G$ be a separable totally disconnected compact group which acts effectively on a connected orientable $n$-gm $M$ over the field $L$.

Suppose that $G$ acts trivially on $H_{c}^{n}(M ; L)$.

(i) If $G$ is finite and the characteristic of $L$ is 0 or prime to the order of $G$, or

(ii) If $G=A_{p}$ and the characteristic of $L \neq p$, or

(iii) If $G$ is an arbitrary totally disconnected group and the characteristic of $L$ is 0 ,

then $M / G$ is an orientable $n$-gm over $L$.

Proof. Let $x^{*} \in M / G$. Suppose $U_{1}^{*}$ is a connected open set containing $x^{*}$. Let $x \in M$ such that $f(x)=x^{*}$, where $f$ denotes the orbit map $f: M \rightarrow M / G$. Let $U_{2}^{*}$ be an open connected set such that $x^{*} \in \bar{U}_{2}{ }^{*} \subset U_{1}^{*}$. Furthermore, by Lemma 3 choose $U_{2}^{*}$ so small that $j^{*}: H_{c}^{r}\left(U_{2}\right) \rightarrow H_{c}^{r}\left(U_{1}\right)$ is trivial, $r \neq n$, where the $U_{i}$ are the components of $f^{-1}\left(U_{i}^{*}\right)$ that contain $x, i=1,2$. Let $G_{1}$ and $G_{2}$ be the maximal open and closed subgroups of $G$ which leave $U_{1}$ and $U_{2}$ invariant, respectively (see Lemma 2). Clearly $G_{2} \subset G_{1}$ and if $G_{2}$ is an invariant subgroup of $G_{1}, G_{1} / G_{2}$ is a finite group.

Consider the commutative diagram

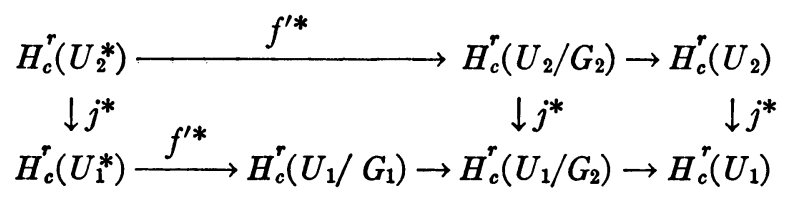

The homomorphisms $f^{*}$ are isomorphisms onto. If $G$ is a finite group the remaining horizontal homomorphisms are isomorphisms into since multiplication in $L$ by a number, (factor of the order of $G$ ), prime to the characteristic of $L$ is an isomorphism (see Lemma 4). Since $j^{*}: H_{c}^{r}\left(U_{2}\right) \rightarrow H_{c}^{r}\left(U_{1}\right)$ is trivial, $r \neq n$, it follows that $j^{*}: H_{c}^{r}\left(U_{2}^{*}\right) \rightarrow H_{c}^{r}\left(U_{1}^{*}\right)$ is trivial. If $G$ satisfies (ii) or (iii) then there exists a sequence of subgroups $G=G_{0} \supset G_{1} \supset G_{2} \supset G_{3} \supset \ldots$ such that $G_{i+1}$ is an invariant open and closed subgroup of $G, G_{i} / G_{i+1}$ is a finite group and $\cap G_{i}$ is the identity, $i \geqq 2$. Thus the spaces $U_{1}$ and $U_{2}$ are the $\operatorname{Inv} \lim U_{1} / G_{i}$ and Inv $\lim U_{2} / G_{i}$, and hence $H_{c}^{+}\left(U_{1}\right)=\operatorname{Dir} \lim H_{c}^{r}\left(U_{1} / G_{i}\right)$ and $H_{c}^{r}\left(U_{2}\right)=\operatorname{Dir} \lim H_{c}^{r}\left(U_{2} / G_{i}\right)$. Furthermore, since the maps from $U_{j} / G_{i}$ to $U_{j} / G_{i-1}(j=1,2$, and all $i)$ are just the orbit maps of the action of the finite group $G_{i-1} / G_{i}$, it follows by the same reasons de- 
scribed above that all the horizontal homomorphisms are isomorphisms into. Consequently, $j^{*}: H_{c}^{\top}\left(U_{2}^{*}\right) \rightarrow H_{c}^{r}\left(U_{1}^{*}\right)$ is trivial, $r \neq n$. Hence the $r$ th local co-Betti number $p^{r}(x ; M / G)=0, r \neq n$.

If $G$ acts trivially on $H_{c}^{n}(M), U$ is an open connected subset of $M$, and if $G^{\prime}$ is any subgroup of $G$ leaving $U$ invariant then $G^{\prime}$ acts trivially on $H_{c}^{n}(U)$. (This follows from the fact that $j^{*}: H_{c}^{n}(U)$ $\rightarrow H_{c}^{n}(M)$ is an isomorphism onto.) Since all horizontal maps in the diagram above are injections and since $H_{c}^{n}\left(U_{2}\right) \approx j^{*} H_{c}^{n}\left(U_{1}\right) \approx L$ it follows that there exists an $i \geqq 2$ such that $H_{c}^{n}\left(U_{2} / G_{i}\right) \approx i^{*} H_{c}^{n}\left(U_{1} / G_{i}\right) \approx L$. It is easy to see from what has been mentioned above that $G_{j} / G_{i}$ acts trivially on $H_{c}^{n}\left(U_{j} / G_{i}\right), j=1,2$. Thus applying Lemma 5 it follows that $H_{c}^{n}\left(U_{2}^{*}\right) \approx i^{*} H_{c}^{n}\left(U_{1}^{*}\right) \approx L$. Observe that this last remark does not depend upon the homomorphism $j^{*}: H_{c}^{s}\left(U_{2}\right) \rightarrow H_{c}^{s}\left(U_{1}\right), s \neq n$. Hence $U_{1}^{*}$ can be chosen to be the entire space $M / G$ and $U_{2}^{*}$ can be chosen to be any connected open subset of $U_{1}^{*}$, when $r=n$. Thus $M / G$ is an orientable $n$-gm over $L$ and this completes the proof.

REMARKs. The assumption that $M$ was orientable and that $G$ acted trivially on $H_{c}^{n}(M)$ were not used to show that $p^{r}\left(x^{*}, M / G\right)$ $=0, r \neq n$. It can be seen by examining the proof that if $M$ is not assumed orientable and/or if $G$ does not act trivially on $H_{c}^{n}(M)$ then $p^{n}\left(x^{*}, M / G\right)=0$ or 1 . Thus the space $M / G$, in this case, would be a space that closely resembles an $n$-gm.

If $M$ were an orientable $n$-gm over $Z$ and the group $G$ did not reverse the orientation of $M$ then $M$ would be an orientable $n$-gm over every field $L$ and $G$ would act trivially on $H_{c}^{n}(M ; L)$. In the case at hand, if $G$ is finite and contains no elements of order 2 then $G$ cannot reverse the orientation of $M$. If $G=A_{p}, p \neq 2$ then $G$ cannot reverse the orientation.

An interesting example occurs when $G=Z_{2}$ and $M=E^{2 n}$. Let $G$ be the reflection of points in $E^{2 n}$ through the origin. This transformation is orientation preserving. The orbit space is an infinite cone over $2 n-1$ real dimensional projective space. The orbit space is an $n$-gm over any field whose characteristic is not 2 but fails to be an $n$-gm over a field whose characteristic is 2 .

In case $G=A_{p}$ acts freely on an $n$-gm $M$ we may strengthen Theorem 1. However in case $L$ is a field whose characteristic is $p$ the result no longer resembles the action of a finite group acting on a manifold.

TheOREM 2. Let $G$ be a p-adic group (respectively a p-adic solenoid $\Sigma_{p}$ ) and suppose that $G$ acts freely on the connected $n-g m M$ over $Z$. Then $M / G$ is an $n$-gm (respectively $(n-1)-g m$ ) over $L$ if $L$ is a field whose characteristic $\neq p$ and an $(n+1)$-gm (respectively an $n-g m)$ over $L$ if $L$ is a field whose characteristic $=p$. 
Proof. Let $G=A_{p}$. Since $M$ is an $n$-gm over $Z$ it is an $n$-gm over every field. Showing that $M / G$ is a generalized manifold is a local problem. By employing the previous lemmas it can be seen that about each point of $M$ one can find a connected orientable open set and an open subgroup of $G$ which leaves the set invariant and does not reverse the orientation. Thus without any loss of generality we may assume that $M$ is an orientable connected $n$-gm and that $G$ does not reverse the orientation of $M$.

Theorem 1 implies the truth of Theorem 2 if the characteristic of $L \neq p$. In case the characteristic of $L=p$ consider the space $M^{\prime}$ $=M \times_{A_{p}} \Sigma_{p}$ (see $\S 2$ of [2]). Since $M$ is orientable and $G$ does not reverse orientation, $M^{\prime}$ is an orientable $(n+1)$-gm over $Z$ and hence over any field of characteristic $p$. The $p$-adic solenoid $G^{\prime}=\Sigma_{p}$ acts freely on $M^{\prime}$ such that $M^{\prime} / G^{\prime}=M / G$. The inverse image $f^{-1}\left(x^{*}\right)$ of any point $x^{*} \in M^{\prime} / G^{\prime}$ is a $p$-adic solenoid. But, $H^{1}\left(\Sigma_{p} ; Z_{p}\right)=0$; hence $f$ is a Vietoris map for every field of characteristic $p$. Thus one may apply Wilder's monotone mapping theorem, [6], and obtain that $M / G$ is an $(n+1)$-gm over every field of characteristic $p$.

In case $G=\Sigma_{p}$ one only needs to consider the action of a $p$-adic subgroup and then the free action of the circle quotient group. This completes the proof.

I know of no example of a space which is an $n$-gm over some field $L$ but whose cohomology dimension with respect to some coefficient domain differs from $n$. It is likely that such an example exists for $n=3$ and the construction of such an example might lead to the long sought for action of a $p$-adic group on a 3-manifold.

As an application we describe the action of a general compact group on a manifold. Suppose that $G$ is a compact group acting effectively on a connected separable finite dimensional (covering dimension) $n$-gm $M$ over $Z$. By theorems of Montgomery-Zippin $G$ is separable and finite dimensional. If $M$ is not orientable then the action of $G$ may be lifted to the orientable double covering of $M$. Thus we may assume that $M$ is orientable. Let us assume that $G$ is connected. There exists a totally disconnected central subgroup $N$ of $G$ such that $G / N$ is a Lie group $K$. Since $G$ is connected $N$ acts trivially on $H_{c}^{*}(M)$ by $\S 2$ of [2]. By Theorem $1 N$ acts effectively on $M$ and the quotient space $M / N$ is an orientable $n$-gm over $L_{0}$. ( $L_{0}$ is a field whose characteristic is 0 .) The Lie group $K$ acts effectively on $M / N$ and its orbit space is homeomorphic to $M / G$. Let $O^{\prime} \subset M / N$ be the open set of $r$-dimensional orbits of the action of $K$ on $M / N$, where $r$ is the maximum dimension of any orbit under the action of $G$. The set $O^{\prime}$ is open connected and dense in $M / N$ because 
$\operatorname{dim}_{L_{0}}\left(M / N-O^{\prime}\right) \leqq n-2$. (This follows readily from [1, Chapter 9, Theorem 2.1]. However, the proof in the reference cited needs to be modified by selecting a circle subgroup instead of a finite group and then using dimensional parity for fixed point sets of circle transformation groups on generalized manifolds over fields of characteristic 0 .) Let $O$ be the inverse image of $O^{\prime}$ under the action of $N$. The set $O$ is the union of a finite number of components say $O_{1}, \cdots, O_{k}$. By Lemma 2, there is an open and closed subgroup $N_{1} \subset N$ such that $O_{i} / N_{1}=O^{\prime}$ and order of $N / N_{1}=k$. Since $N$ acts effectively on $M, N$ acts effectively on $O$. Thus, if $N$ is not a finite group (in case $G$ is not a Lie group), then $N_{1}$ cannot act effectively as a finite group on each $O_{i}$. In this case, we may as well assume $N_{1}$ is not a finite group and acts effectively on $O_{1}$. Since $O_{1}$ is an orientable $n$-gm over $Z, N_{1}$ cannot contain arbitrarily small finite subgroups and therefore $N_{1}$ must contain a $p$-adic group $A_{p}$, for some prime $p$. By Theorem 3 of [2] $\operatorname{dim}_{Z} O_{1} / A_{p}=n+2$. Since light proper maps cannot lower the cohomology dimension, it follows that $\operatorname{dim}_{Z} O^{\prime} \geqq n+2$ in case $N$ is not a finite group.

Let $x \in O^{\prime}$ and $S_{x}$ be a slice at $x$. There exists an open Euclidean $r$-cell $C^{r}$ such that $C^{r} \times S_{x}$ is an open neighborhood of $x$ by the slice theorem of Montgomery-Yang. The stability group at $x, K_{x}$, acts on the slice $S_{x}$ effectively as a finite group and hence $\operatorname{dim}_{Z} S_{x}=\operatorname{dim}_{Z} S_{x} / K_{x}$. Note that $S_{x} / K_{x}$ is an open neighborhood of the image of $x$ by the orbit map induced by the action of $K$. Furthermore, since $C^{r}$ is an $r$-cell, $\operatorname{dim}_{Z} C^{r}+\operatorname{dim}_{Z} S_{x}=\operatorname{dim}_{Z} C^{r} \times S_{x}$.

Suppose that at $x \operatorname{dim}_{Z} O^{\prime} \geqq n+2$, if $N$ is not finite. Therefore, $\operatorname{dim}_{z} S_{x}=\operatorname{dim}_{z} S_{x} / K_{x} \geqq n-r+2$, which proves (ii) of the Corollary if $G$ is connected.

Note that $S_{x}$ is an orientable $(n-r)$-gm over $L_{0}$ by [4, Theorem 6]. Thus if $r \geqq n-2, S_{x}$ is a locally Euclidean manifold of dimension $\leqq n-r$. This implies that $G$ must be a Lie group.

In case $G$ is not connected, let $G_{0}$ be the component of the identity. Then $G / G_{0}=N$, a totally disconnected group. If $G_{0}$ is not a Lie group then the argument above suffices. If $G_{0}$ is a Lie group, the set of principal orbits $O$ of the action $G_{0}$ on $M$ is an open dense connected subset of $M$. Moreover, it is a locally trivial fiber space and its orbit space $O / G_{0}$ is an $(n-r)$-gm over $Z$ by [4, Theorem 6$]$. The totally disconnected group acts effectively on $O / G_{0}$ and cannot contain arbitrarily small finite subgroups. Thus if $N$ is not finite (in case $G$ is not a Lie group) it must contain a $p$-adic subgroup. The corollary now follows easily. 


\section{REFERENCES}

1. A. Borel, Seminar on transformation groups, Annals of Mathematics Studies No. 46, Princeton, 1960.

2. G. E. Bredon, Frank Raymond, R. F. Williams, p-adic groups of transformations, to appear in Trans. Amer. Math. Soc.

3. D. Montgomery and L. Zippin, Topological transformation groups, New YorkLondon, Interscience, 1955.

4. Frank Raymond, Union and separation theorems for generalized manifolds with boundary, Michigan Math. J. vol. 7 (1960) pp. 7-21.

5. G. T. Whyburn, Analytic topology, Amer. Math. Soc. Colloquim Publications, vol. $28,1942$.

6. R. L. Wilder, Monotone mappings of manifolds. II, Michigan Math. J. vol. 5 (1958) pp. 19-23.

7. C. T. Yang, p-adic transformation groups, Michigan Math. J. vol. 7 (1960) pp. 201-218.

The Institute for Advanced Study

\section{AN EXTENSION OF BANACH'S CONTRACTION PRINCIPLE}

\section{MICHAEL EDELSTEIN}

1. Let $X$ be a metric space and $f$ a mapping of $X$ into itself; $f$ will be said to be a globally contractive mapping if the condition

$$
d(f(p), f(q))<\lambda d(p, q)
$$

with constant $\lambda, 0 \leqq \lambda<1$, holds for every $p, q \in X$. A well known theorem of Banach states:

If $X$ is a complete metric space and $f$ is a globally contractive mapping of $X$ into itself then there exists a unique point $\xi$ such that $f(\xi)=\xi$.

2. It is natural to ask whether the theorem (referred to as Banach's contraction principle) could be modified so as to be valid when condition (1) is assumed to hold for sufficiently close points only. To be more specific we introduce the following notions:

2.1. A mapping $f$ of $X$ into itself is said to be locally contractive if for every $x \in X$ there exist $\epsilon$ and $\lambda(\epsilon>0,0 \leqq \lambda<1)$, which may depend on $x$, such that:

$$
p, q \in S(x, \epsilon)=\{y \mid d(x, y)<\epsilon\} \quad \text { implies (1). }
$$

2.2. A mapping $f$ of $X$ into itself is said to be $(\epsilon, \lambda)$-uniformly

Received by the editors March 25, 1960. 